\title{
Criminologie
}

\section{Du côté des filles et des femmes, leur délinquance, leur criminalité}

\section{Renée Collette-Carrière et Louise Langelier-Biron}

Volume 16, numéro 2, 1983

Les femmes et la justice pénale

URI : https://id.erudit.org/iderudit/017179ar

DOI : https://doi.org/10.7202/017179ar

Aller au sommaire du numéro

Éditeur(s)

Les Presses de l'Université de Montréal

ISSN

0316-0041 (imprimé)

1492-1367 (numérique)

Découvrir la revue

Citer cet article

Collette-Carrière, R. \& Langelier-Biron, L. (1983). Du côté des filles et des femmes, leur délinquance, leur criminalité. Criminologie, 16(2), 27-45.

https://doi.org/10.7202/017179ar d'utilisation que vous pouvez consulter en ligne.

https://apropos.erudit.org/fr/usagers/politique-dutilisation/ 


\section{DU CÔTÉ DES FILLES ET DES FEMMES LEUR DÉLINQUANCE, LEUR CRIMINALITÉ Renée Collette-Carrière* Louise Langelier-Biron**}

\section{CONTEXTE THÉORIQUE}

Une des premières constatations scientifiques qui alimente les propos au sujet de la délinquance des femmes et des filles réside sans nul doute dans le fait que la participation de ces dernières à la criminalité est faible sur le plan de l'ampleur et sur celui de la gravité des actes. En 1899, Lombroso affirmait: "Toutes les statistiques s'accordent pour démontrer que la proportion des crimes est beaucoup moindre chez les fermmes que chez les hommes" (p. 214). Le discours de la littérature scientifique a fort longtemps qualifié cette différence de naturelle pour des raisons tantôt biologiques, tantôt psychologiques ou sociologiques. La criminalité des femmes et des filles a été expliquée à partir d'éléments issus d'une conception bien particulière des rôles féminins et masculins. Qualifiée d'insignifiante par comparaison statistique avec celle des hommes, cette criminalité a souvent été décrite comme n'étant qu'une activité prostitutionnelle ou celle d'une cleptomane. Ces pôles explicatifs relevaient et relèvent encore d'une vision étroite et souvent erronée de la réalité. Notre propos ne réside pas dans une déconstruction complète de cette image stéréotypée de la crimilalité dite féminine. Cependant, il nous semble opportun dans un premier temps de faire le point quant à l'évolution de la pensée criminologique sur ce phénomène et ultérieurement, de jeter un regard sur les faits statistiques et leur évolution.

Les quinze dernières années ont vu apparaître une littérature abondante au sujet de la déviance des filles et des femmes. Cette abondance et l'émergence d'un discours novateur nous paraîssent fortement tributaires de la montée du féminisme. Il s'agit d'un effet positif de ce mouvement vertement critiqué par ailleurs. Des femmes ont jeté un regard sévère à la fois sur la littérature scientifique concernant leur déviance et aussi sur la réalité telle que perceptible et perçue. nente.

*Chargée de cours à l'École de criminologie et à la Faculté d'éducation perma-

**Professeur à l’École de criminologie, Université de Montréal. 
Antérieurement, la majorité des auteurs, la plupart masculins, avaient axé leurs explications à partir de la dualité «madone ou prostituée». Les thèses observées alors, à cause de cette vision de la femme, tournent autour de notions telles la passivité naturelle chez la femme ou sa moralité supérieure, la différence biologique entre les sexes et la naturelle infériorité féminine, la sexualité, la prostitution, etc. Ces dernières années, des thèmes nouveaux ont été mis en place, jetant un regard différent sur ce phénomène. Ces interventions peuvent parfois être rattachées à un courant criminologique identifié, mais ce n'est pas toujours le cas. Nous tenterons donc de faire le point en présentant ces thèmes et certains de leurs initiateurs. Cette revue des quinze dernières années ne se prétend pas exhaustive, mais elle se veut un point de redépart, nous l'espérons, vers la formulation d'une pensée de plus en plus créatrice et articulée. L'apparition des différents thèmes n'est pas toujours chronologique dans notre description, mais nous croyons que nous rejoignons la majorité des idées émises.

Plusieurs auteurs ont dénoncé le paramètre utilisé pour mesurer la criminalité féminine. C'est Frances Heidensohn (1968) qui déclare que ce paramètre ne peut mesurer adéquatement la criminalité des femmes et des filles puisque les normes sur lesquellesil se base sont masculines; on tente donc de mesurer des réalités différentes avec la même échelle. De concert avec Campbell (1977), elle dénonce aussi la sexualisation de leur criminalité. Elle démontre que cette dernière est acquisitive. D'autres auteurs soutiennent cette constatation: Alder, 1975; Simon, 1975; Crites, 1976; Smart, 1976; Bertrand, 1979; Datesman et Scarpitti, 1980, etc. Dans la même veine, dans son ouvrage intitulé Sisters in Crime, Freda Adler (1975) établit que le fait qu'il y ait peu de femmes délinquantes prouve que l'on hésite à reconnaître la criminalité des femmes. C'est un peu comme si les représentants de l'ordre public avaient une mission spéciale envers le sexe féminin : reproduire les normes et valeurs du groupe dominant en l'occurrence des hommes. Le principe du parens patriae, qu'il soit adopté par le père de la jeune fille, par son mari, par le policier ou encore par le juge, sous-entend que la fille est faible, qu'elle a besoin de support et que finalement on doit la guider.

Ces mêmes auteurs ont pour la plupart aussi procédé à une dénonciation des théories biologiques et du biais sexiste qu'elles véhiculent.

C'est Adler (1975) qui a introduit l'idée de la dangerosité du féminisme : en se libérant, les femmes vont devenir plus criminelles 
et cette criminalité sera de plus en plus violente. D'autres ont emboîté le pas (Austin, 1982). L'idée d'une augmentation de la criminalité classique découlant de l'émancipation de la femme dans la société n'est cependant pas partagée par un certain nombre d'auteurs, dont Crites (1976), Smart (1979), Campbell (1981) et Norland et al. (1981). On invoque alors l'argument que la couche de population qui est touchée par les statistiques est celle qui est la plus démunie et la moins susceptible d'être happée par un courant féministe quel qu'il soit. Plusieurs ont plutôt opté pour une analyse critique ou féministe face aux explications et à la réalité même de la criminalité (Klein, 1973; Smart, 1976; Bertrand, 1979; Plenska, 1980;). Certaines notions sont alors apparues : socialisation différentielle selon le sexe, structure différentielle des opportunités illégitimes (Hotiman Bustamante, 1973), dénonciation des stéréotypes à l'endroit des femmes lesquelles pourraient amener une certaine complaisance de la part des autorités policières et judiciaires et par le fait même une possible contre-réaction. Cette idée lait aussi référence aux notions d'attitudes et de réactions sociales différentielles (Simon, 1975; Cir tes, 1976; Smart, 1976; Bertrand, 1979; Warren. 1981). Des liens ont été élaborés entre les deux concepts suivants : criminalité féminine' 't condition féminine (Smart, 1976; Bertrand, 1979; Adler et Simun, 1979; Feinmen, 1980). La notion d'intervention différentielle selon le sexe est analysée dans ce système jusqu'au niveau des programmes correctionnels et des ressources postpénales (Giallombardo, 1966; Chandler, 1973; Brodsky, 1975; Crites, 1976; Adler et Simon, 1979; Berzins et Collette-Carrière, 1979; Berzins et Cooper, 1982;).

Du côté des filles, Chesney-Lind (1977) analyse, en lonction des mineurs, la réaction des tribunaux et conclut qu'elle s'inspire directement de la réaction qu'ont "normalement» les parents des jeunes filles. Ainsi, comme de bons parents, policiers, juges et agences sociales répondent différemment aux garçons et aux filles pour la bonne raison qu'il est plus grave pour une lille d'être délinquimte que pour un garçon d'être délinquant.

De même, s'il se dégage un certain consensus quant à l'importance de la famille pour la fille, il se peut qu'une attention toute particulière soit donnée aux jeunes filles dont la lamille ne répond pas aux normes d'une famille conventionnelle et ce, dans des proportions plus grandes que pour les garçons. Cela traduirait la croyance (vraie ou fausse) selon laquelle les jeunes filles ont davantage besoin que les garçons d'un milieu familial adéquat. Ces dernières remarques nous amènent à considérer un point de vue plus critique qui pose le problème plus en dehors de la fille-même. 
La nécessité d'inventorier de nouvelles approches (Smart, 1976; Bertrand, 1979) liées à une perspective historique d'une part et à un souci de recherche transculturelle d'autre part (Adler et Simon, 1979; Adler, 1981) émerge de plus en plus. Certains secteurs de la déviance et la place qu'y occupent les femmes et les filles deviennent un lieu de questionnement tel le terrorisme (Cooper in : Adler et Simon, 1979), telles certaines réalités pouvant être définies par quelques-uns comme des alternatives à la criminalité pour les femmes (Chesler, 1972; Bertrand, 1979;) ou l'appartenance des filles à des bandes (Steffensmeier, 1980;).

Le mode d'analyse et les conclusions des auteurs cités ne sont pas tous les mêmes mais ils ont en commun ceci qu'ils ont remplacé une conception statique par une approche plus dynamique mettant en cause l'univers social et culturel qui nous entoure. Ceci étant dit que nous réservent les données actuelles par rapport à la criminalité des adolescentes et des femmes?

\section{LA DÉLINQUANCE DES ADOLESCENTES}

Il est une donnée qui ressort avec force et constance. Il s'agit du fait que les filles commettent moins d'acte délinquants que les garçons. Quel que soit le pays, la méthode de cueillette des données, l'âge, la période, le milieu socio-économique, l'écart subsiste. Il convient bien sûr de qualifier cette différence et par conséquent de la nuancer puisque comme le soulignent Hindelang et al. (1981) «la corrélation entre le sexe et la criminalité varie considérablement selon le temps et le lieu, par ailleurs les différences selon le sexe demeurent importantes dans toutes les données officielles disponibles actuellement» (p. 137)'.

Les pages qui suivent sont consacrées à la description du phénomène de la délinquance chez l'adolescente. Il sera question de sa nature, de sa diversité et de son évolution. De plus, s'il est intéressant de situer ces données dans un contexte local, il n'en demeure pas moins utile de procéder à des comparaisons entre divers milieux géographiques.

\section{LA DÉLINQUANCE AILLEURS}

Aussi, il convient de rappeler les plus récents développements à ce chapitre. Ainsi, Giordano (1978) rapporte que les filles de race

1. The correlation between sex and criminality varies markedly over place and time, vet sex differences remain pronounced in all official data momently available. 
blanche de son échantillon commettent plus d'actes délinquants quand elles fréquentent surtout un groupe mixte que quand elles se tiennent principalement avec des filles. Il reste à préciser pourquoi le fait qu'il y ait présence de garçons augmente la délinquance. Est-ce simplement un phénomène de stimulation de groupe ou est-ce que la fille acquiert une certaine expertise au contact de garçons plus expérimentés? L'auteur émet l'hypothèse que c'est plutôt la deuxième interprétation qui prévaut. Ceci lui semble d'autant plus plausible que les adolescentes s'avèrent plus sensibles à l'approbation ou à la désapprobation des amis du même sexe.

Le même auteur associé à Cernkovich publie en 1979 une étude basée sur l'analyse comparative de garçons et de filles qui ont répondu à un questionnaire autorévélé.

Leur étude récente confirme que de fait plus de garçons que de filles répondent positivement à chacun des 36 actes délinquants proposés. Le rapport garçon/fille est de 2,18 , une valeur plus faible selon les auteurs que celle qui est rapportée par Hindelang (1971) soit 2,56 pour la délinquance révélée et 3,72 pour la délinquance officielle. De plus, quand la moyenne d'implication est calculée pour chacun des actes délinquants, près de $28 \%$ des différences entre les sexes ressortent comme non significatives et les auteurs observent une certaine analogie entre les conduites délinquantes des garçons et des filles.

Ce sont d'ailleurs ces résultats qui font dire aux auteurs en 1979 qu'il est impérieux :

d'examiner les secteurs particulièrement touchés par l'évolution de la délinquance des filles et la nature de la réaction à ces conduites(p. 60).

afin d'alimenter les théories dejjà formulées.

Le souhait formulé par Cernkovich et Giordano (1979) semble trouver une réponse dans l'étude des Steffensmeier (1980). Ceux-ci rapportent, en effet, que l'analyse des arrestations et l'étude des bandes d'adolescentes révèlent très peu d'augmentation dans les délits violents ou reliés aux activités de bandes. Ceci veut dire qu'il n'y a pas de rapprochement entre garçons et filles pour ce qui est d'une délinquance violente grave. Par ailleurs, les statistiques provenant de Uniform Crime Reports indiquent une augmentation des délits de fugue, des petits vols et des violations de la loi sur l'alcool. Les statistiques judiciaires et les données provenant de délinquance révélée confirment ces résultats à la différence qu'elles soulignent un accroissement dans l'usage de la marijuana. Á partir de ces résultats, 
les auteurs concluent que rien dans ces changements ne démontre une modification dans des conduites reliées à un nouveau rôle pour la fille.

À la même période, soit en 1980, le Conseil de l'Europe publie trois rapports sur la criminalité des femmes, ceux de Einsele, Fontanesi et Goodman. À l'exception de l'étude de Fontanesi, ces documents font une brève allusion à la délinquance des mineures. Ainsi Einsele (1980) affirme au sujet des condamnations en Allemagne que :

l'augmentation qu'on observe chez les jeunes filles, aussi importante qu'elle puisse paraître proportionnellement, demeure en valeur absolue bien inférieure à celle qu'on constate chez les garçons (p. 17).

Par rapport aux infractions, l'auteur souligne que pour les jeunes filles, l'accroissement est relativement faible de 1963 à 1978 et pour les crimes violents et pour les infractions contre les biens.

Pour sa part, Goodman (1980) analyse les données en Angleterre et au Pays de Galles et rapporte que :

la délinquance féminine présente un phénomène inquiétant: l'augmentation croissante des jeunes femmes de moins de 21 ans (p. 28).

Il convient de souligner que Goodman ne fait état que des délits graves, c'est-à-dire de ceux que nous appellerions actes criminels par opposition aux infractions. À cet égard, l'auteur souligne que pour les vols qualifiés et les cambriolages, le taux réel le plus élevé et l'augmentation la plus forte se situent chez les filles de 14 à 17 ans.

L'étude extrêmement minutieuse des mesures de la délinquance effectuée par Hindelang $e t$ al. (1981) consacre un chapitre aux différences selon le sexe. En fonction de ce qui vient d'être rapporté ci-haut, les auteurs attirent l'attention sur le fait que la réduction de l'écart entre garçons et filles au niveau des données of ficielles ne se vérifie pas de façon aussi tangible dans les données qui proviennent d'un questionnaire autorévélé.

Hindelang et al. (1981) analysent, dans un premier temps, les données officielles parues aux États-Unis et observent qu'en 1976 (après une augmentation régulière depuis 1960) un quart des arrestations des filles ont trait aux petits vols. Pour les garçons, ces délits reflètent un cinquième des arrestations. Les différences les plus importantes apparaissent dans la catégorie de vols avec effraction et des fugues. Les premiers constituent $13 \%$ de la délinquance des garçons et $31 \%$ de la délinquance des filles. Les derniers présentent une 
configuration inverse soit plus de $23 \%$ chez les filles et moins de $5 \%$ chez les garçons.

11 est également intéressant de souligner que les quatorze délits plus fréquemment commis par les garçons sont les mêmes que ceux qui sont perpétrés par les filles. Ainsi, les adolescentes américaines présentent une configuration d'actes délinquants analogue à celle des garçons.

Dans un deuxième temps, les auteurs ont entrepris de recenser quelques 25 études basées sur la délinquance révélée. Les diverses analyses effectuées révèlent que les comparaisons avec les données officielles sont difficiles puisque les énoncés ne mesurent pas nécessairement les mêmes conduites. Il n'en demeure pas moins qu'une analyse selon la variété des actes ou la fréquence entraîne des résultats similaires. Ainsi, il existe des différences selon le sexe, quelle que soit la façon de mesurer la délinquance révélée pour toutes les conduites, à l'exception de la drogue. Par contre, Hindelang et al. (1981) ne manquent pas de nous mettre en garde contre une interprétation trop hâtive de ces résultats. Des variations dans le rapport fille/garçon sur des énoncés spéciliques tendent à illustrer le fail que des comparaisons globales peuvent nous entraîner sur de fausses pistes. D'une part, elles masquent des écarts importants selon le type de délit et d'autre part, elles donnent l'impression d'une certaine inconsistance avec les données officielles.

Cette difficulté est quelques peu contournée par Canter (1982) qui inclut une très grande variété d'actes délinquants dans son étude sur les différences selon le sexe dans la délinquance révélée. Les résultats révèlent que les différences selon le sexe sont demeurées constantes entre 1967 et 1977 , qu'elles sont néanmoins présentes à travers les délits spécifiques et les mesures plus globales de la délinquance. Ces différences apparaissent toutefois de faible amplitude et une fois de plus la configuration des délits rapportés ressort de façon non spécifique selon le sexe.

Ce bref tour d'horizon des données provenant d'autres contextes géographiques s'arrête avec l'étude longitudinale de Suzanne Ageton (1982). Considérant les liens par trop faciles qu'on a pu établir entre l'émancipation de la femme et l'augmentation de ses délits (Adler, 1975; Austin, 1982), les résultats rapportés par Ageton s'avèrent très intéressants. En effet, elle a pu établir que les jeunes filles de 15-17 ans en 1980 sont significativement moins impliquées dans la délinquance que celles du même âge en 1976. De plus, les données recueillies sur une période de cinq ans indiquent une diminution ou 
une stabilité de la délinquance à mesure que la fille traverse sa période d'adolescence.

Les données qui viennent d'être transmises permettent de mieux situer les informations à caractère plus local dont il sera maintenant question.

\section{LA DÉl.INQUANCE ICI}

\section{Les données officielles}

L'exercice de l'analyse des données statistiques relatives à la fille en matière socio-légale s'avère extrêmement frustrant à plus d'un égard. Dans certains cas, il y a une absence totale de données récentes, par exemple au niveau des établissements de protection. Ainsi, depuis 1973, Statistique Canada ne compile plus ce type d'information. Dans d'autres cas, les données ne sont pas disponibles selon le sexe. C'est le cas des jeunes jugés hors cours et rapportés dans les statistiques policières. Enfin, il arrive que ces changements au niveau de l'application de la loi entraîne des incongruités dans la façon de rapporter les faits. L'année 1979 constitue un exemple patent de ce problème. L'application de la loi 24 au Québec a de fait modifié le dénombrement des cas dans les statistiques policières de façon telle que la majorité des sujets ont été inscrits sous la rubrique «hors cours». II devient donc quasi impossible de procéder à des séries chronologiques à partir des statistiques policières.

Ces difficultés étant connues, il est plus facile de rapporter les données officielles sans craindre de leur donner une portée qu'elles n'ont pas.

La délinquance officielle telle que rapportée par les statistiques policières (Québec, 1981) indique une certaine constance dans le rapport filles/garçons à tout le moins pour l'ensemble des délits et ce, depuis quelques années. Ainsi, en 1975 le rapport est de 1:6 et en 1981, il est de 1:7.

En regardant de plus près la nature des délits, on remarque en 1981 une concentration des filles $(44,6 \%)$ sous la rubrique «Autres statuts provinciaux». Cette rubrique, en plus de rassembler les délits reliés à l'alcool, englobe la loi 24 de protection de la jeunesse, loi qui touche aussi bien les cas de protection que de délinquance. En deuxième place, ce sont les vols de $200 \$$ ou moins $(20,3 \%)$ qui ressortent suivis de loin $(10,8 \%)$ par les vols par effraction. Cette répartition des délits revêt une certaine constance à travers les années. 
Il est intéressant de remarquer, chez les garçons, un ordre quelque peu différent où les vols par effraction $(39,5 \%)$ dominent la série. En deuxième et troisième places se trouvent les vols de $200 \$$ ou moins $(17,1 \%)$ et les autres infractions au code criminel avec $12,4 \%$.

Il convient également de souligner que le rapport filles/garcons est relativement constant sur les rubriques ci-mentionnées à l'exception d'une seule, les vols par eftraction. En effet, une analyse des statistiques policières au Québec révèle qu'entre 1975, 1978 el 1981 , le ratio demeure à peu près le même, soit 1,3:1 pour les autres statuts provinciaux, 1:5 pour les vols de $200 \$$ ou moins, 1:18 pour les autres infractions au code criminel. Par contre, les vols par effraction sont en progression pour les filles, progression illustréc par le fait qu'en 1975 le rapport filles/garçons est de 1:58, en 1978, il est de $1: 41$ et en 1981, il passe à 1:29. Ainsi la distance ent re l'implicaltion des garçons et des filles pour ce délit particulier s'amenuise progressivement.

En 1979, une analyse des données of ficielles (Ciagnon et Biron) faisait clairement ressortir que les filles commetlent beaucoup moins de délits que les garçons mais que par ailleurs la majorité de leurs délits respectifs touchent une délinquance acquisitive. Les donnces récentes provenant du Québec font état d'une proportion d'implication dans ce type de délinquance de $74 \%$ pour les garçons et de $43 \%$ pour les filles. Ces faits conservent leur actualité. Il y était également mentionné que les filles se retrouvent en proportion plus grande que les garçons à la rubrique «Autres statuts provinciaux» el cela se confirme toujours. De plus, les auteurs observaient une augmentation dans les délits contre les biens, augmentation qui suit son cours à travers les données présentées.

Cette vision de la criminalité des adolescentes gagne toutefois à être complétée par la présentation de données autoconlessécs qui découlent de différentes recherches entreprises.

\section{Les données autoconfessécs}

À partir de données recueillies auprès d'adolescentes de 12 à 18 ans de l'île de Montréal, il est possible d'établir que $28,9 \%$ répondent négativement à 32 actes inscrits au code criminel (Biron et al., 1980). En comparant ce pourcentage avec celui des garcons $(9,8 \%$, s'il s'ensuit que trois fois plus de filles que de garçons n'ont jamais commis de délits de cet ordre au cours des douze derniers mois. Cette différence ne s'observe pas au niveau de la délinquance dite statu- 
taire, c'est-à-dire celle qui découle de l'âge des sujets (fugue, absentéisme scolaire, alcool, etc.). En effet, un pourcentage à peu près égal soit $87,7 \%$ et $93,9 \%$ ressort de la comparaison entre filles et garçons. Il convient également de souligner qu'au niveau de la souséchelle de vol, échelle qui s'apparente par son contenu aux catégories de vol dont il est fait mention dans les statistiques ci-haut mentionnées, le rapport entre filles et garçons qui commettent ces actes est de 1:2 (Fréchette et LeBlanc, 1979).

Pour ce qui est des populations contrastées, soit les adolescentes en milieu libre et en milieu fermé, une recherche effectuée en 1979 (Gagnon et al.) permet d'affirmer que la délinquance des filles en centres d'accueil est nettement supérieure à celles des filles en milieu libre. De fait, elle est à toutes fins pratiques comparable à celle des garçons en milieu libre.

Une deuxième remarque vise l'évolution de la délinquance chez les adolescentes prises à diverses périodes. Une seule question a été posée à des adolescentes de 14 à 18 ans en 1976 et en 1982, il s'agit de la fugue. Alors que ce groupe d'âge répondait «jamais» dans une proportion de $96,7 \%$ en 1976, six ans plus tard, un groupe du même âge répond à la négative dans une proportion de $83,5 \%$. Il est certes difficile d'extrapoler, à partir d'une seule réponse, une quelconque tendance. Il reste néanmoins, qu'au niveau de la fugue, les données autoconfessées indiquent une augmentation.

\section{RÉFLEXION SUR I,ES FAITS}

S'il est indéniable que les filles commettent moins de délits que les garçons et qu'elles sont appréhendées surtout pour des motifs relevant plutôt de conduites problématiques que d'actes criminels, il n'en demeure pas moins que la délinquance autoconfessée met en évidence le fait que les filles commettent plus de délits qu'il n'y paraît officiellement. Cela est également vrai pour les garçons. Par contre, si on tient compte du rapport filles/garçons, il s'ensuit que le ratio est beaucoup plus faible au niveau de la délinquance autoconfessée que de la délinquance officielle.

En raison même des problèmes signalés au sujet de l'entrée en vigueur de la loi 24 au Québec, il devient extrêmement difficile d'évaluer l'évolution de la délinquance. Quant à la délinquance autoconfessée, il n'existe pas d'études longitudinales récentes qui permettent d'établir de façon systématique qu'effectivement les filles commettent plus ou moins d'actes délinquants que leurs conscurs en ont commis dans le passé. 
ll convient, à la lumière de ces faits, de faire ressortir deux aspects qui effectivement posent problème. Le premier tient à une certaine redéfinition des conduites de la fille qui s'opère à divers niveaux d'intervention. Le second porte sur le besoin d'explorer la conduite des adolescentes à partir d'un univers autre que celui de la légalité pénale.

En effet, même si la fille commet moins d'actes délinquants que le garçon, l'écart entre les données of ficielles et les données autoconfessées pour ce qui a trait au rapport fille/garçon révèle que ceux qu'elle commet sont récupérés sous l'appellation d'un besoin de protection. Comme les mentalités sont plus lentes à changer que les lois, l'application de la nouvelle loi 24 n'a pas sensiblement modifié cette pratique.

Le second aspect relève plus du domaine hypothétique. Il serait intéressant de vérifier auprès des adolescentes qu'est-ce qui constitue une infraction. C'est là tout le problème de la définition du crime qui ne sera certes pas abordée ici. Il n'en demeure pas moins que la fille est particulièrement sensible à tout ce qui touche les relations interpersonnelles ${ }^{2}$. Sa déviance ne s'exprime peut-être pas dans le cadre du crime traditionnellement défini mais pourrait prendre d'autres formes découlant du mal que les personnes peuvent se faire entre elles à d'autres niveaux.

\section{LA CRIMINALITÉ DES FEMMES}

Où en est rendue la criminalité des femmes statistiquement? Quelle est son évolution? S'est-elle développée d'une manière de plus en plus violente? Voilà quelques questions auxquelles nous tenterons de répondre.

\section{AUX ÉTATS-UNIS}

Comme dans la plupart des pays industrialisés, la criminalité des Américaines a effectivement augmenté. Datesman et Scarpitti (1980) rapportent qu'en 1977, la proportion des femmes parmi les arrestations totales a augmenté d'un peu plus de $5 \%$ par rapport à 1960, c'est-à-dire qu'elle est passée de $10,7 \%$ à $16 \%$. En ce qui concerne les crimes majeurs', l'augmentation est forte et ce sont les cri-

2. Voir à cet effet l'excellent livre de Carol (iilligan (1982).

3. Le FBI public les données des arrestations dans un rapport intitulé Uniform (rime Reports qui touche les deux catégories suivantes : "index crime" et "non-index trime" que nous avons traduit crimes majeurs et crimes mineurs bien arbitrairement. I a premiere categorie comprend sept crimes : meurtre, viol, assaul grave, vol qualifié, vol par elfraction, vol simple, vol d'atto; la seconde concerne 22 autres types de crime. 
mes contre la propriété qui font monter la représentation fëminine ( $10 \%$ en 1960 et $22,4 \%$ en 1977). Quant aux crimes violents, la proportion demeure stable ( $10 \%$ en 1960 et $10,5 \%$ en 1977). Dans les crimes contre la propriété, rappelons que ce sont surtout les vols simples (larceny) qui sont responsables de la plus grande partic de l'augmentation. Quant aux crimes mineurs, il y a eu hausse aussi et elle se situe dans les délits reliés à la fraude. La criminalité masculine augmente aussi mais selon les auteurs, au niveau adulte, le taux d'arrestation féminine croît plus rapidement. Nous n'élaborerons pas davantage sur la situation américaine.

\section{AU CANADA ET AU QUÉBEC}

Certains chercheurs dont Bertrand (1979) ont démontré que la criminalité féminine a augmenté au Canada à un rythme un peu supérieur à celui des hommes. Adams (1978) affirme qu' 'en 1965, la police a inculpé dix fois plus d'hommes que de femmes et qu'en 1975, le rapport n'était que 8 pour 1 " (p. 25). En vertu du code criminel, 86,4\% des personnes accusées en 1975, étaient de seve masculin (adultes et mineurs) et 13,6\% de sexe féminin. En 1978, ces proportions étaient respectivement 85,2 et $\left.14,8 \%{ }^{4}\right)$. En ce qui concerne les adultes pour l'année $1981^{\mathrm{s}}$, les femmes représentent $10,9 \%$ de l'ensemble des personnes accusées en vertu du code criminel en comparaison de $11,1 \%$ en 1975. Pour l'ensemble des lois, la proportion des femmes est de $9,6 \%$ en 1981, elle était de $9 \%$ en 1975 (voir en annexe tableau 1). Au Québec, 62,8\% des contrevenants sont des adultes masculins, $9,5 \%$ féminins, $24,5 \%$ des mineurs masculins et $3,2 \%$ féminins en $1981^{\circ}$. La situation québécoise en ce qui concerne les personnes accusées est donc comparable à la sit uation canadienne en général.

L'activité des femmes dans la criminalité a augmenté dans les crimes contre la propriété. Ces derniers constiluent plus des deux tiers de leurs activités crimineiles (voir tableau 2). En 1975, cette catégorie représentait $64,9 \%$ des crimes féminins et en 1981, il s'agit de $69,3 \%$. Statistique Canada remarque que de 1977 à 1981, le pourcentage de femmes inculpées de crimes contre la propriété s'est élevé de $45,4 \%$ tandis que chez les hommes, cette hausse a été de

4. Voir Baril et Collette-Carrière (1981), p. 74. rie : jeunes.

5. Statistique Canada ne sépare pius les filles des garçons et n'a qu’une carçgo-

6. Chiffres calculés à part ir des données trouvées dans Statistiques 1981: (ri minalité et application des règlements de la circulation au Québec, Quéhec, r. 41. 
$37 \%{ }^{7}$. Du côté des crimes de violence contre les personnes, on note peu de changement (voir tableau 2). La criminalité de violence n'a pas vraiment augmenté chez les femmes.

De quels crimes sont accusées les femmes? De crimes contre la propriété et en 1981, elles se situent principalement dans les vols de 200 s et moins $(32,3 \%$ des infracteurs adultes dans cette catégorie) et dans les affaires relatives aux fraudes $(24,1 \%$ des infracteurs adultes). Le tableau 3 permet de bien saisir la situation comparative pour 1975-1978 et 1981. Celle-ci montre une image stable dans les crimes de violence contre les personnes et certaines augmentations au niveau des crimes contre la propriété (vol de plus de $200 \$$, fraude). D'ailleurs, dans les vols de plus de $200 \$$ et de moins de $200 \$$, la sous-catégorie vol à l'étalage regroupe $42,8 \%$ et de $44,8 \%$ de femmes respectivement parmi les adultes actifs dans chacune de ces activités en $1981^{*}$. Globalement, parmi les accusés adultes en vertu du code criminel, la proportion de femmes est passée de $14 \%$ en 1975 à $15 \%$ en 1978 et à $15,1 \%$ en 1981. Au Québec, le tableau 4 nous fait voir un portrait semblable.

Le délit de prostitution requierait une analyse particulière. Le chiffre a baissé, entre autres raisons, suite à des jugements de la Cour suprême et il y aurait lieu d'en faire l'objet d'arguments séparés. Ce n'est cependant pas l'objectif de cet article".

Au niveau des condamnations, nous ne pouvons aller plus loin que Adams (1978) ou Bertrand (1979). Les statistiques des tribunaux que nous avons regardées (1978) ne donnent pas de données selon le sexe. La proportion des femmes n'a jamais dépassé $16 \%$ et c'est pour des crimes contre la propriété que les femmes sont condamnées. Ce sont ceux qui ont le plus augmenté. Ces tendances semblent se maintenir. Les sentences données aux femmes comptent beaucoup d'amendes, de probation et moins d'emprisonnement. Par contre, il apparaît que lorsqu'il y a décision, depuis ces dernières années, d'imposer une sentence de plus de 2 ans, l'écart, quant à la durée de l'incarcération, tend à décroître entre les hommes et les femmes ${ }^{10}$. 1981, p. 43.

7. Statistiques de la criminalité et des règlements de la circulation (police),

8. Proportions calculées à partir des données des Statistiques de la criminalité et des règlements de la circulation (police), Tableau 2, 1981.

9. Voir Baril et Collette-Carrière (1981), p. 75. De plus, plusieurs auteurs ont discuté de cette question dans différents arricles.

10. Adams (1978), p. 39. 
Au chapitre de l'emprisonnement, au Canada, les femmes représentent environ $6 \%$ de la population carcérale totale. En 1979, la proportion des femmes en milieu pénitentiaire fédéral était de $1,7 \%{ }^{11}$, Berzins et Cooper (1982) parlaient de 200 détenues pour 10000 détenus, soit $2 \%$ et en mars 129 détenues sur 11261 personnes incarcérées dans un établissement fédéral, soit une proportion de $1,1 \% 0^{12}$.

Au Québec, on note une augmentation de la population carcérale féminine. Celle-ci s'explique en grande partie par le fait que les Québécoises purgeant des sentences de plus de 2 ans, puissent «faire leur temps» dans les prisons provinciales surtout à la Maison Tanguay. La proportion des femmes, dans l'ensemble des prisons de la province était de $4 \%$ en $1977^{1 .}$ et en 1981 , elle est de $5,7 \%$; ce qui signifie 1903 femmes et 31427 hommes $^{14}$. La moyenne quotidienne a aussi augmenté : de 79 en 1975, elle est passée à 113 en 1977, 114 en 1979 et 133 en 1981 (selon un calcul approximatif). La durée des sentences ayant augmenté par l'arrivée des sentencées fédérales et les délits jugés graves étant punis plus sévèrement, il est normal que cette moyenne ait aussi augmenté.

\section{CONCLUSION}

Faire le point au sujet de la criminalité des filles et des femmes, tel était l'objectif de cet article. Le point sur les différents aspects entourant le phénomène tant dans la littérature que dans les faits. II nous apparaît très important que toute cette question soit étudiée dans une perspective d'ensemble. Les différents thèmes élaborés depuis ces dernières années ressortent comme des jalons posés dans ce sens. Ils ne sont donc en soi qu'une partie d'un tout à venir. Les faits statistiques ne dévoilent pas toute la réalité non plus. Il existe face à ce phénomène une nécessité d'aller plus loin par des recherches à partir de l'acquis certes mais dans un esprit ouvrant la voie à une théorisation plus adaptée à la réalité des filles et des femmes de notre époque et à la criminologie contemporaine.

11. Berzins et Collette-Carrière (1979).

12. Service Correctionnel Canada (1983), p. 14 Chiffres tirés du Rapport hebdomadaire sur la population de détenus S.C.C., Ottawa, pour la semaine finissant le 15 mars 1983.

13. Berzins et Collette-Carrière (1979), p. 19.

14. Statistiques correctionnelles québécoises (1981), p. 24. 
TABLEAU 1

Répartition pourcentuelle des personnes ac'usées par la police, selon le sexe et le groupe d'áge (adulte et juvénile) pour toutes les lois, Canada, 1975 et 1981

\begin{tabular}{|c|c|c|c|c|c|}
\hline & Année & Hommes & Femmes & Jeunes & Total \\
\hline Code criminel & $\begin{array}{l}1975 \\
1981\end{array}$ & $\begin{array}{l}68,23 \\
60,7\end{array}$ & $\begin{array}{l}11,13 \\
10,9\end{array}$ & $\begin{array}{l}20,64 \\
28,4\end{array}$ & $\begin{array}{l}100,(0) \\
100,00\end{array}$ \\
\hline $\begin{array}{l}\text { Lois fédérales touchant } \\
\text { les drogues }\end{array}$ & $\begin{array}{l}1975 \\
1981\end{array}$ & $\begin{array}{l}85,24 \\
84,8\end{array}$ & $\begin{array}{l}9,23 \\
9,0\end{array}$ & $\begin{array}{l}5.53 \\
6,2\end{array}$ & $\begin{array}{l}100.00 \\
100.00\end{array}$ \\
\hline Autres lois fédérales & $\begin{array}{l}1975 \\
1981\end{array}$ & $\begin{array}{l}85,51 \\
77,0\end{array}$ & $\begin{array}{l}7,20 \\
12,0\end{array}$ & $\begin{array}{l}7,29 \\
11,0\end{array}$ & $\begin{array}{l}100,0(0) \\
100,00\end{array}$ \\
\hline Lois provinciales & $\begin{array}{l}1975 \\
1981\end{array}$ & $\begin{array}{l}89,48 \\
86,8\end{array}$ & $\begin{array}{l}6,28 \\
7,4\end{array}$ & $\begin{array}{l}4,24 \\
5,8\end{array}$ & $\begin{array}{l}100,00 \\
100,00\end{array}$ \\
\hline Lois municipales & $\begin{array}{l}1975 \\
1981\end{array}$ & $\begin{array}{l}83.66 \\
77,7\end{array}$ & $\begin{array}{l}10,57 \\
14,2\end{array}$ & $\begin{array}{l}5,77 \\
8,1\end{array}$ & $\begin{array}{l}300,(0) \\
100 .(00\end{array}$ \\
\hline Pourcentage moyen & $\begin{array}{l}1975 \\
1981\end{array}$ & $\begin{array}{l}78,68 \\
72,62\end{array}$ & $\begin{array}{l}8.99 \\
9,61\end{array}$ & $\begin{array}{l}12,33 \\
17,77\end{array}$ & $\begin{array}{l}100,00) \\
100,00\end{array}$ \\
\hline
\end{tabular}

Source: Statistiques de la criminalité et des réglements de la circulation (police). Canada. Pour l'année 1975 : voir Bertrand (1979), p. 56, 1ableall 3; cependant, nous avons additionné les pourtentages des garcons et des litles, car les statistiques de 1981 ne font pas la distinction. Pour lannée 1981, les pourcentages ont été tirés ou calculés par l'autcuré de cet article.

\section{TABLEAU 2}

Proportion des adultes accusés au Code criminet seulomen. selon le sexe et par calégories d'offenses.

Canata, 1975-1978-1981

\begin{tabular}{|c|c|c|c|}
\hline & Annec & Honmes & Femules \\
\hline & & $\%$ & $\pi_{0}$ \\
\hline $\begin{array}{l}\text { Crimes de violence v's } \\
\text { personnes }\end{array}$ & $\begin{array}{l}1975 \\
1978 \\
1981\end{array}$ & $\begin{array}{l}16.76 \\
15,69 \\
14,54\end{array}$ & $\begin{array}{l}8.98 \\
8.38 \\
8.55\end{array}$ \\
\hline Crimes vs propriéte & $\begin{array}{l}1975 \\
1978 \\
1981\end{array}$ & $\begin{array}{l}48.18 \\
47.17 \\
50.46\end{array}$ & $\begin{array}{l}64,91 \\
67,83 \\
69,25\end{array}$ \\
\hline Autres crimes & $\begin{array}{l}1975 \\
1978 \\
1981\end{array}$ & $\begin{array}{l}35,06 \\
37,14 \\
34,98\end{array}$ & $\begin{array}{l}26.11 \\
23.79 \\
22.19\end{array}$ \\
\hline Total pour chaque annéc & & 100,00 & $100,0 \mathrm{k})$ \\
\hline
\end{tabular}

Source: Stastiques de la criminalité et des règlements de la circulation (polive). Tableau 2 (1-2-3-4), Canada, 1975-1978-1981. Annéc 1975: voir Bertrind (1979), p. 57, tableau 4. Années 1978-1981 : calculées par l'aut cure de cet article. 
TABLEAU 3

Proportion de femmes parmi les adultes accusés pour les principales infractions et familles d'infraction au Code criminel, Canuda, 1975-1978-1981

$\%$ de Femmes

\begin{tabular}{|c|c|c|c|}
\hline & 1975 & 1978 & 1981 \\
\hline Homicide ( $y$ inclus les tentatives) & 12,3 & 12,1 & 12,4 \\
\hline Crimes sexuels & 1,2 & 0,6 & 1,3 \\
\hline Lésions corporelles (voie de lajt) & 8,9 & 9,7 & 10,7 \\
\hline Vol qualifié & 6,6 & 6,7 & 7,2 \\
\hline $\begin{array}{l}\text { Total : Crimes de violence contre } \\
\text { les personnes }\end{array}$ & 8,0 & 8,6 & 9.5 \\
\hline Pénétrer par effraction & 3,4 & 4,0 & 4,2 \\
\hline Vol de véhicule moteur & 3,8 & 4,4 & 5,2 \\
\hline Vol de plus de $200 \$$ & 11,7 & 9,1 & 13,9 \\
\hline Vol de $200 \$$ et moins & 31,0 & 33,8 & 32,3 \\
\hline Recel (avoir en sa possession) & 10,4 & 12,0 & 11,8 \\
\hline Fraudes & 19,0 & 23,4 & 24,1 \\
\hline Total : Crimes contre la propriété & 18,0 & 20,2 & 19,7 \\
\hline Prostitution & 77,3 & 47,3 & 46,4 \\
\hline Jeux et paris & 5,1 & 3,7 & 5,7 \\
\hline Armes offensives & 4,9 & 5,4 & 6,4 \\
\hline Autres of fenses au Code criminel & 9,0 & 10,0 & 10,1 \\
\hline Total : Autres crimes & 10,8 & 10,1 & 10,2 \\
\hline Total : Code criminel & 14,02 & 15,02 & 15,15 \\
\hline
\end{tabular}

Source: Pourcentages calculés à partir du total des adultes (hommes et femmes) pour chaque rubrique à partir des chiffres de Statistiques de la criminalité et des règlements de la circulation (police), tableau 2. Canada. 1975-19781981.

TABLEAU 4

Répartition pourcentuelle des adultes accusés au Code criminel, aux lois et aux règlements seton le sexe et par catégories d'offenses, Québec, 1981

\begin{tabular}{lrrr}
\hline & Hommes & Femmes & Total \\
\hline $\begin{array}{l}\text { Crimes avec violence } \\
(8,52 \% \text { de toute la criminalité) }\end{array}$ & 94,31 & 5,69 & 100,00 \\
$\begin{array}{l}\text { Crimes contre la propriéte } \\
(42,67 \% \text { de toute la criminalité) }\end{array}$ & 82,38 & 17,62 & 100,00 \\
$\begin{array}{l}\text { Autres crimes Code criminel } \\
(22,90 \% \text { de toute la criminalité) }\end{array}$ & 89,12 & 10,88 & 100,00 \\
$\begin{array}{l}\text { Infraction lois et règlements } \\
(25,92 \% \text { de toute la criminalité) }\end{array}$ & 87,19 & 12,81 & 100,00 \\
\hline
\end{tabular}

Source: Statistique 1981 : Criminalité et application des règlements de la circulation au Québec. Service de polices municipaux, Sûreté du Québec et Giendamerie royale du Canada. Québec, p. 40. 
BIBLIOGRAPHIE

ADAMS, Susan Gillis (1978), La délinquante : étude statistique. Ottawa : ministère du Solliciteur général.

ADLER, Freda (1975). Sisters in Crime. New York : McGraw-Hill.

ADLER, Freda, SIMON, Rita James (Ed.) (1979). The Criminology of Deviant Women, Boston : Houghton Mifflin Co.

ADLER, Freda, (Ed.) (1981). The Incidence of Female Criminality in the Contemporary World. New York : New York University Press.

AGETON, S. S. (1982). The Dynamic of Female Dilinquency, 1976-1980, Document inédit. Boulder, Colorado : Behavioral Research Institue.

AUSTIN, Roy L. (1982). «Woman's Liberation and Increases in Minor, Major and Occupational Offenses». Criminology, 20, 3-4, 407-430.

BARIL, Micheline, Renée COLLETTE-CARRIËRE, (1981). "Femmes criminelles, femmes victimes : nouvelles perspectives d'action." Cahiers scientifiques et pratiques, Congrès 1981 de l'Association professionnelle des criminologues du Québec, vol. 2, novembre.

BERTRAND, Marie-Andrée (1979). La femme et le crime. Montréal : L'Aurore.

BERZINS, Lorraine, Renée COLLETTE-CARRIÈRE (1979). «La fernme en prison, un inconvénient social?» Santé mentale au Québec, IV, 2.

BERZINS, Lorraine, Sheelagh COOPER, (1982). «The Political Economy of Correctional Planning for Women : The Case of the Bankrupt Bureaucracy", Revue canadienne de criminologie/Canadien Journal of Criminology, 24, 4, 399-416.

BIRON, L., R., GAGNON, M. LEBLANC, (1980). La délinquance des filles. Cahier $n^{\circ} 3$, Groupe de recherche sur l'inadaptation juvénile, Université de Montréal.

BRODSKY, Annette M. (Ed.) (1975). The Female Offender, Beverly Hills, Ca : Sage.

CAMPBELL, A (1977). "What Makes a Girl Turn Crime?" New Society, 27, January, 172-173.

CAMPBELL, A. (1981). Girl Delinquents. Oxford : Basic Blackwell.

CANTER, R. J. (1982). "Sex Differences in Self-Report Delinquency». Criminology, 20 3.4, 373-393.

CERNKOVICH, S.A., P.C. GIORDANO, (1979). "A Comparative Analysis of Male and Female Delinquency". The sociological quaterly, 20, winter, 131-145.

CHANDLER, Edna Walker (1973). Women in prison. Indianapolis : The BobbsMerrill.

CHESLER, Phyllis, (1972). Les femmes et la folie. Paris: Payot, 1975.

CHESNEY-LIND, M. (1977). «Judicial paternalism and the female status of fender», crime and Delinquency, 23, 2, 121-130.

CRITES, Laura (Ed.) (1976). The Female Offender. Lexington, Mass, : Lexington Books.

DATESMAN, Susan K., Frank R. SCARPITTI, (Ed.) (1980). Women, Crime and Justice. New York : Oxford University Press.

EINSELE, H. (1980). La criminalité féminine en République fédérale d'Allemagne. Strasbourg: Conseil de l'Europe. 
FEINMAN, Clarice (1980). Women in the Criminal Justice System. New York : Praeger.

FONTANESI, M. (1980). Lacriminalité des femmes en ltalie. Strasbourg : Conseil de l'Europe.

FRECHETTE, M., M., LEBLANC (1979). La délinquance cachée à Montréal, Cahier $n^{\circ} 1$, Groupe de recherche sur l'inadaptation juvénile, Université de Montréal.

GIALLOMBARDO, Rose (1966). Society of Women, a Study of a Women's Prison. New York : John Wiley.

GAGNON, R., L. BIRON (1979). Les filles marginalisées : perspective statistique. Rapport $\mathrm{n}^{\circ} 1$, Groupe de recherche sur l'inadaptation juvénile, Université de Montréal.

GAGNON, R., L. BIRON, M.A. BERTRAND (1979). Aspirations et délinquance révélée chez les adolescentes. Rapport $\mathrm{n}^{\circ} 3$, Groupe de recherche sur l'inadaptation juvénile, Université de Montréal.

GILLJGAN, C. (1982). In a Different Voice. Cambridge : Harvard University Press.

GIORDANO, P.C. (1978). "Girls, Guys and Gangs : The Changing Socail Context of Female Delinquency«. The Journal of criminal law and criminology, 69, 1, 126-132.

GIORDANO, P.C., S.A. CERNKOVICH (1979). Changing Patterns of female Delinquency, Research proposal. Unpublished.

GOODMAN, N. (1980). La Délinquance féminine. Evvolution en Angleterre et au Pays de Gailes, 1963-1977. Strasbourg : Conseil de l'Europe.

HEIDENSOHN, F. (1968). "The Deviance of Women : A Critique and an Inquiry". British Journal of sociology, 192, 160-176.

HINDELANG, M.J. (1971). Age, Sex and the Versatility of Delinquent Involve. ments. Social problems, 18, 522-535.

HINDELANG, M.J. T., HIRSCHI, J.G. WEIS (1981). Measuring Dilinquency. Beverly Hills : Sage.

HOFFMAN-BUSTAMANTE, Dale (1973). "The Nature of Female Criminality". Issues in criminology, 8, 2, 117-136.

KLEIN, Dorie (1973). «The Etiology of Female Crime : A Review of the Litcrature". Issues in Criminology, 8, 2, 3-30.

LOMBroSO, C. (1899). Le crime, causes et remèdes, Paris : Scleicher Frères Editeurs.

NORLAND, S., R.C. WESSEL, N. SHOVER (1981). "Masculinity and Delinquency). Criminology, 19, 3, 421-433.

PLENSKA, Danuta (1980). «L a criminalité féminine en Pologne». Revue canadienne de criminologie, $22,4,464-475$.

QUÉBEC (1981). Caractéristique des jeunes contrevenants. Québec 1974-1980, Direction de la recherche et du développement, Direction générale de la sécurité publique, ministère de la Justice, décembre.

SER VICES CORRECTIONNELS CANADA (1983). Recueil de données concernant les services correctionnels canadiens. Ottawa : Solliciteur général du Canada.

SIMON, Rita James (1975). Women and Crime. Lexington, Mass. : Lexington Books. 
SMART, Carole (1976). Women, Crime and Criminology, a Feminist Critique. Londres : Routledge et Kegan Paul.

SMART, C. (1979). «The New Female Criminal : Reality or Myth?» British Journal of criminology, 19, 1, 50-59.

STATISTIQUES DE LA CRIMINALITE ET DE L'APPLICATION DES RËGLEMENTS DE LA CIRCULATION. Statistique Canada : Centre canadien de la statistique juridique. Ministère des Approvisionnements et Services, Ottawa, Canada, 1975, 1978, 1981.

STATISTIQUES, 1981. CRIMINALITÉ ET APPLICATION DES RĖGLEMENTS DE LA CIRCULATION AU QUÉBEC. Gouvernement du Québec : ministère de la Justice, Direction générale de la sécurité publique.

STATISTIQUES CORRECTIONNELLES QUÉBÉCOISES (1981). Gouvernement du Québec, ministère de la Justice, Direction générale de la probation et de la détention.

STEFFENSMEIER, D.J., STEFFENSMEIER, R.H. (1980). Trends in Female Delinquency. Criminology, 18, 1, 62-85.

WARREN, Marguerite Q. (Ed.) (1981).Comparing Female and Male Offenders. Beverly Hills, Ca. : Sage. 